\title{
Real-time frequency-encoded spatiotemporal focusing
}

\author{
X. Wei ${ }^{1 \dagger}$, Y. Shen ${ }^{1,2 \dagger}$, J. C. Jing ${ }^{1 \dagger}$, A. S. Hemphill ${ }^{1}$, C. Yang ${ }^{3}$, S. Xu ${ }^{3}$, Z. Yang ${ }^{3 *}$, and L. V. Wang ${ }^{* *}$ \\ ${ }^{I}$ Caltech Optical Imaging Laboratory, Andrew and Peggy Cherng Department of Medical Engineering, Department of Electrical Engineering, \\ California Institute of Technology, 1200 East California Boulevard, Mail Code 138-78, Pasadena, California 91125, USA. \\ ${ }^{2}$ Present address: School of Electronics and Information Technology, Sun Yat-sen University, Guangzhou 510006, China. \\ ${ }^{3}$ State Key Laboratory of Luminescent Materials and Devices, Guangdong Engineering Technology Research and Development Center of Special \\ Optical Fiber Materials and Devices, Guangdong Provincial Key Laboratory of Fiber Laser Materials and Applied Techniques, South China \\ University of Technology, Guangzhou 510640, China. \\ These authors contributed equally to this work. \\ *yangzm@scut.edu.cnorLVW@caltech.edu
}

\begin{abstract}
We present a novel real-time frequency-encoded spatiotemporal (FEST) focusing technology using a programmable two-dimensional optical frequency comb. This technique enables, for the first time, simultaneous spatial and temporal focusing at microseconds through thick scattering media. (C) 2020 The Author(s)

OCIS codes: (140.3518) Lasers, frequency modulated; (030.6140) Speckle; (170.7160) Ultrafast technology
\end{abstract}

\section{Introduction}

In the fields of biophotonic imaging, optogenetics, and laser surgery, focusing deep into living tissues is essential for precise diagnosis, manipulation, and treatment [1]. The microscopic inhomogeneity inherent in biological objects, however, randomizes incident light and limits high-resolution biomedical applications to small depths, usually $<1$ $\mathrm{mm}$ in soft tissues [2], preventing whole-body animal and human studies. To overcome this obstacle, the concept of wavefront shaping [3-5] has been developed and applied to focus photons deep into or through scattering media. The existing wavefront shaping techniques can be mainly classified into three categories: feedbackbased wavefront shaping [3], transmission matrix measurement [4], and optical phase conjugation (OPC) [5]. The former two approaches obtain the optimal wavefront through an iterative process, and their runtimes are usually long, conventionally up to a few hours. MEMS-based spatial light modulators (SLMs) associated with field programmable gate array (FPGA) electronics and one-dimensional GLV phase modulators have recently been employed to reduce system runtimes [6,7], but with limited number of control modes. In contrast, OPC approaches determine the optimal wavefront in a few shots using two-dimensional (2D) cameras [8]. However, a considerable amount of time is required to read out the data from the camera sensor arrays. Up until, the state-of-the-art OPC system still takes a few milliseconds to measure the optimal wavefront and transfer the data to the computer or processor [9]. Here we present a novel technology, termed frequency-encoded spatiotemporal (FEST) focusing, that can generate a sharp spatiotemporal focus through thick dynamic scattering media at microsecond scales.

\section{Experimental setup and results}

Figure 1a illustrates the principle of the real-time FEST focusing technology. The backbone of the FEST focusing system is a programmable $2 \mathrm{D}$ optical frequency comb (2D-OFC) that is generated from a homebuilt CW laser with an ultrafine linewidth of $1.8 \mathrm{kHz}$. The 2D-OFC is employed for both high-speed wavefront measurements and spatiotemporal focusing, through parallel optical beating and localized mode locking, respectively. As shown in Fig. 1a, a single input laser beam is acousto-optically diffracted into a 2D-OFC beam array by driving two orthogonal acousto-optic deflectors (AODx,y) with specially designed multitone radiofrequency (RF) signals (e.g., Fig. 1b showing a $23 \times 24$ beam array). Each sub-beam of the 2 D-OFC beam array has a unique frequency and angle. The 2D-OFC beam array then illuminates a high-speed SLM (HS-SLM), where the beam array is pixelmatched with the HS-SLM to perform independent 2D phase compensation for spatiotemporal focusing. A scattering medium (SM) is placed after the HS-SLM, where the 2D-OFC beam array is randomized into a time-variant multifrequency speckle pattern. The time-variant speckle light is then combined with a reference beam at a different optical frequency through a beam splitter (BS), and beats at a high-speed photodetector (PD). The beating signal, e.g., Fig. 1c, is then digitized by a high-speed data acquisition (DAQ) card and sent to a graphics processing unit (GPU) for fast phase retrieval. To this end, the beating signal is first converted to the frequency domain through fast Fourier transformation (FFT), resulting in $\mathrm{M} \times \mathrm{N}$ distinct peaks in the frequency spectrum, e.g., Fig. 1d. The phases of all frequencies are calculated by the four-quadrant inverse tangent operation, followed by a subtraction of the calibration term (if necessary). The phase sequence is then shaped into a $2 \mathrm{D}$ conjugated phase map according to the pixel matrix (Fig. 1b), and addressed to the HS-SLM, after which a spatiotemporal focus is generated, as shown in Fig. 1e, which is generated by using a $35 \times 36$ beam array. The complete FEST focusing system is controlled by a customized $\mathrm{C}++$ program with low latency that 
operates in a continuous streaming mode. Thanks to the unprecedented speed of FEST technology, we have successfully applied it to demonstrate, for the first time, spatiotemporal bright-field and fluorescence imaging through highly scattering biological tissues, as well as focusing through an in vivo mouse ear, all at microsecond scales (the results are not shown here due to the length limit).

\section{(a)}

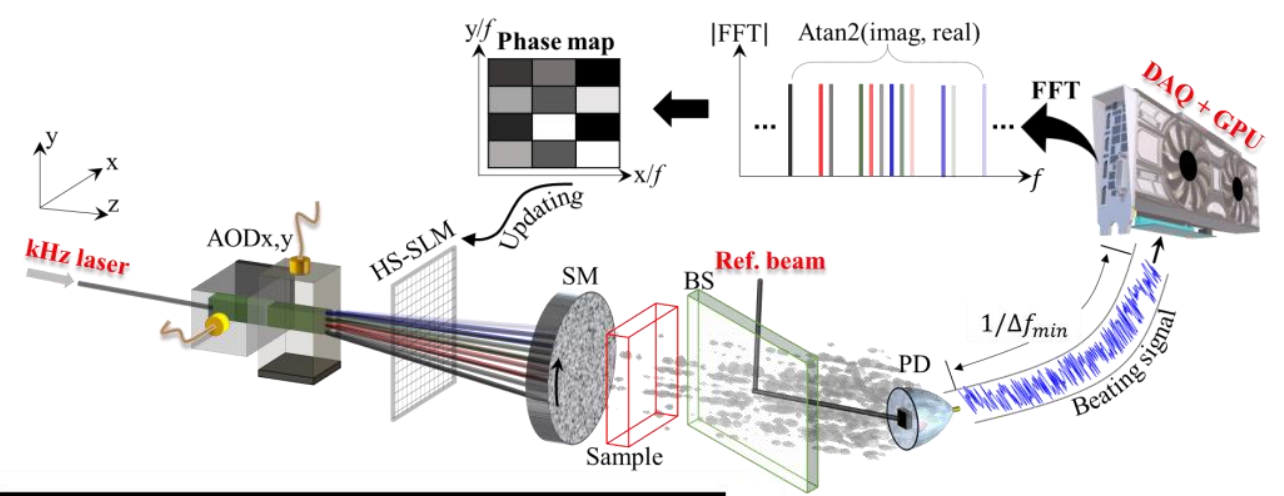

\section{(b)}

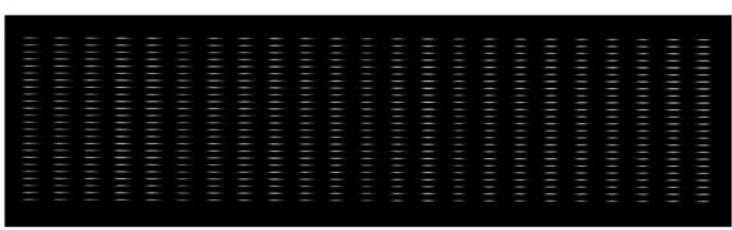

(c)

(d)
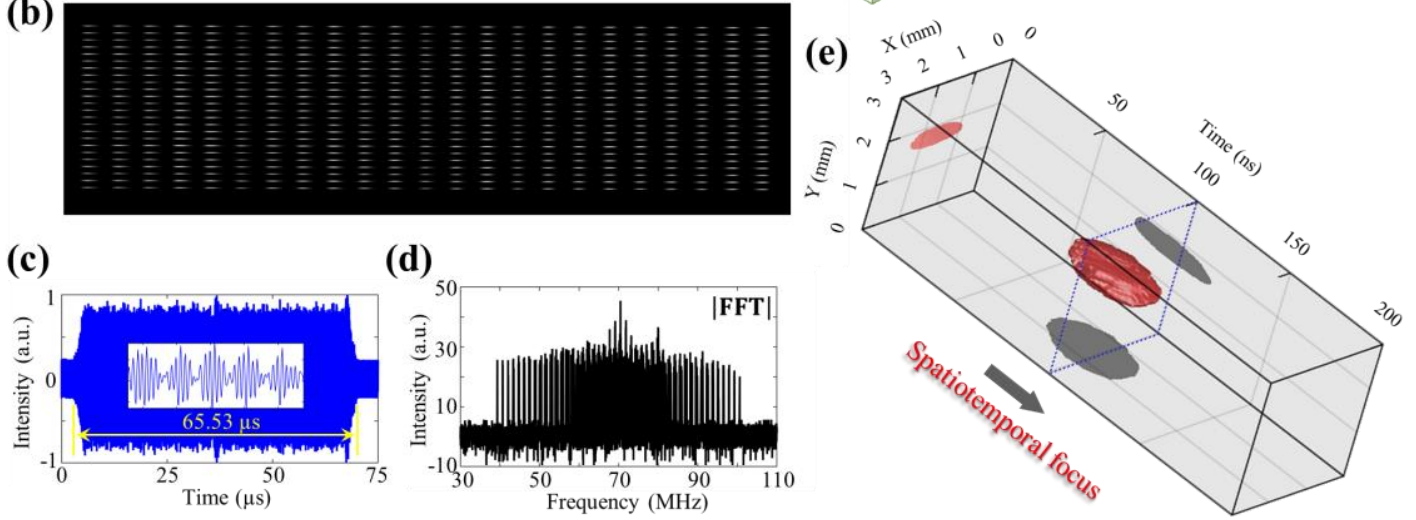

Fig. 1. (a) The principle of the real-time FEST focusing technology. (b) A typical 2D-OFC beam array that has a spot number of $23 \times 24$. (c) A typical beating signal. The inset shows small features of the beating signal. (d) The frequency spectrum of the beating signal after performing fast Fourier transformation. (e) The spatiotemporal focus spot after FEST focusing.

\section{Conclusion}

In conclusion, we have reported a novel real-time wavefront shaping technology - frequency-encoded spatiotemporal focusing using a programmable 2D ultrafine optical frequency comb - that enables, for the first time, simultaneous spatial and temporal (3D) focusing at microsecond scales through thick dynamic scattering media. We anticipate that this work opens a new avenue for high-speed wavefront shaping with the potential to revolutionize the field of photonics.

\section{Acknowledgment}

This work was partially supported by Local Innovative and Research Teams Project of Guangdong Pearl River Talents Program No. 2017BT01X137, China; US National Institutes of Health (NIH) Grants No. DP1 EB016986 (NIH Director's Pioneer Award), and R01 CA186567 (NIH Director's Transformative Research Award).

\section{References}

[1] S. H. Yun and S. J. J. Kwok, Nat. Biomed. Eng. 1, 0008 (2017).

[2] A. P. Mosk, A. Lagendijk, G. Lerosey, M. Fink, Nat. Photon. 6, 283-292 (2012).

[3] I. M. Vellekoop, A. P. Mosk, Opt. Lett. 32, 2309-2311 (2007).

[4] S. M. Popoff, G. Lerosey, R. Carminati, M. Fink, A. C. Boccara, and S. Gigan, Phys. Rev. Lett. 104, 100601 (2010).

[5] Z. Yaqoob, D. Psaltis, M. S. Feld, C. Yang, Nat. Photon. 2, 110-115 (2008).

[6] B. Blochet, L. Bourdieu, S. Gigan, Opt. Lett. 42, 4994-4997 (2017).

[7] O. Tzang, E. Niv, S. Singh, S. Labouesse, G. Myatt, and R. Piestun, Nat. Photon. 33, 788-793 (2019).

[8] M. Cui, C. Yang, Opt. Express 18, 3444-3455 (2010).

[9] D. Wang, E. H. Zhou, J. Brake, H. Ruan, M. Jang, and C. Yang, Optica 2, 728-735 (2015). 